\title{
On the mKdV-Liouville hierarchy and its self-similarity reduction
}

\author{
Danilo V. Ruy \\ Instituto de Física Teórica-UNESP, Rua Dr Bento Teobaldo Ferraz 271, Bloco II, 01140-070, São \\ Paulo, Brazil
}

\begin{abstract}
Integrable mixed models have been used as a generalization of traditional integrable models. However, a map from a traditional integrable model to a mixed integrable model is not well understood yet. Here, it is studied the relation between the mKdV-Liouville hierarchy and the mKdV hierarchy by employing an extended version of the modified truncation approach. This paper shows some solutions for the mKdV-Liouville hierarchy constructed from the soliton solutions of the mKdV hierarchy. The last section deals with the possibility of define new transcendental functions from the self-similarity reduction of the mKdV-Liouville hierarchy.
\end{abstract}

Keywords: mKdV-sinh-Gordon, mKdV-Liouville, mKdV, modified truncation approach, Painlevé, transcendental function

2010 MSC: 37K10, 37K35, 37K40

\section{Introduction}

The mKdV equation has been widely studied in the last decades and possess some well know solutions [1, 2, 3, 4. A generalization of the mKdV equation which combines the $\mathrm{mKdV}$ and the sinh-Gordon equations was proposed in [5] as a mixed integrable model. Later, this mixed model showed to be suitable for describing few-optical-cycles pulse in transparent media [6]. The generalized $m K d V$-sinh-Gordon hierarchy [7] is an mixed model which include the mixed mKdV-sinh-Gordon $(\delta(t)=-\beta(t))$ and the mixed mKdV-Liouville hierarchies $(\delta(t)=0)$, namely

$$
\epsilon_{0}(t) \frac{\partial}{\partial x}\left(\frac{\partial}{\partial x}+y_{x}\right) \mathcal{L}_{n}\left[x ; y_{x x}-\frac{1}{2} y_{x}^{2}\right]+y_{x t}+\beta(t) e^{y}+\delta(t) e^{-y}=0
$$

where $\mathcal{L}_{n}[x ; u]$ is the Lenard recurrence relation, i.e.

$$
\frac{\partial}{\partial x} \mathcal{L}_{n+1}[x ; u]=\left(\frac{\partial^{3}}{\partial x^{3}}+2 u \frac{\partial}{\partial x}+u_{x}\right) \mathcal{L}_{n}[x ; u], \quad \mathcal{L}_{0}[x ; u]=1,
$$

such that the first equation of the hierarchy is

$$
\epsilon_{0}(t)\left(y_{x x x x}-\frac{3}{2} y_{x}^{2} y_{x x}\right)+y_{x t}+\beta(t) e^{y}+\delta(t) e^{-y}=0
$$

Email address: daniloruy@ift.unesp.br (Danilo V. Ruy) 
It was shown in [7] that the self-similarity reduction of (2) yields Kudryashov's equation [8. Such equation passes the necessary condition for absence of movable branches points, called Painlevé property, and it can reduce to two Painlevé equation by appropriate choices of the parameters. The Painlevé equations are second-order nonlinear ODEs (ordinary differential equations) which define new transcendental functions [9] and it has motivated many studies in higher order ODEs [7, 8, 10, 11, 12, 13, 14, 15, 16, 17, 18, 19, 20]. Due the connection with two Painlevé equations, Kudryashov's equation was conjectured as a possibility of defining a new transcendental function [8, 21]. The representation of the solutions of (1) and its self-similarity reduction in terms of a simpler hierarchy is not well understood yet. In this paper, we study this relation in the particular case of the mKdV-Liouville hierarchy.

The modified truncation approach was introduced in [22] as a technique to find autoBäcklund transformations for an hierarchy of ODEs. Here, we employ an extended version of this approach in order to obtain one transformation from soliton solutions for the $\mathrm{mKdV}$ hierarchy to the mKdV-Liouville hierarchy. Besides, we obtain a transformation from the PII hierarchy to a particular case of the Kudryashov's hierarchy.

\section{Transformations for the mKdV-Liouville hierarchy}

Let us consider the hierarchy (1) with $\delta(t)=0$ and $\epsilon_{0}(t) \neq 0$. Due to theorem 2 of appendix, we can choose $\epsilon_{0}(t)=1$ without lost of generality. So we consider the mKdV-Liouville hierarchy as

$$
\frac{\partial}{\partial x}\left(\frac{\partial}{\partial x}+y_{x}\right) \mathcal{L}_{n}\left[x ; y_{x x}-\frac{1}{2} y_{x}^{2}\right]+y_{x t}+\beta(t) e^{y}=0
$$

In order to transform $\mathcal{L}_{n}\left[x ; y_{x x}-\frac{1}{2} y_{x}^{2}\right]$ in a Möbius invariant element, we do the transformation

$$
y=\ln \left[g(t) \sigma_{x}\right],
$$

where $g(t)$ is a arbitrary function. Thus, the hierarchy (3) becomes

$$
\frac{\partial}{\partial x}\left(\frac{\partial}{\partial x}+\frac{\sigma_{x x}}{\sigma_{x}}\right) \mathcal{L}_{n}[x ; S(\sigma)]+\frac{d}{d t}\left(\frac{\sigma_{x x}}{\sigma_{x}}\right)+\beta(t) g(t) \sigma_{x}=0
$$

where $S(\sigma)$ is the Schwarzian derivative, i.e.

$$
S(\sigma)=\frac{d}{d x}\left(\frac{\sigma_{x x}}{\sigma_{x}}\right)-\frac{1}{2}\left(\frac{\sigma_{x x}}{\sigma_{x}}\right)^{2}
$$

By doing the Möbius transformation $\sigma=-1 / \phi$ and defining $\tilde{y}=\ln \left[g(t) \phi_{x}\right]$, we obtain the relation

$$
y=\ln \left[g(t) \frac{\phi_{x}}{\phi^{2}}\right]=\ln \left[g(t) \phi_{x}\right]-2 \ln \phi \equiv \tilde{y}-2 \ln \phi
$$

and the hierarchy (5) is transformed into

$$
\frac{\partial}{\partial x}\left(\frac{\partial}{\partial x}+\frac{\phi_{x x}}{\phi_{x}}-2 \frac{\phi_{x}}{\phi}\right) \mathcal{L}_{n}[x ; S(\phi)]+\frac{d}{d t}\left(\frac{\phi_{x x}}{\phi_{x}}-2 \frac{\phi_{x}}{\phi}\right)+\beta(t) g(t) \frac{\phi_{x}}{\phi^{2}}=0,
$$


which it can be rewrite as

$$
\frac{\partial}{\partial x}\left(\frac{\partial}{\partial x}+\tilde{y}_{x}-2 \frac{\phi_{x}}{\phi}\right) \mathcal{L}_{n}\left[x ; \tilde{y}_{x x}-\frac{1}{2} \tilde{y}_{x}^{2}\right]+\frac{d}{d t}\left(\tilde{y}_{x}-2 \frac{\phi_{x}}{\phi}\right)+\beta(t) g(t) \frac{\phi_{x}}{\phi^{2}}=0
$$

Two cases are considered in the following subsections by using equation (8), i. e. the partial differential equation (3) and a self-similarity reduction of it.

\subsection{From a soliton solution of the $m K d V$ hierarchy to a $m K d V$-Liouville solution}

Observe that $g(t)$ only appears multiplying $\beta(t)$ in (8). As we can redefine $\beta(t)$ using theorem 2 of appendix, we can assume $g(t)=1$ without lost of generality.

If $\tilde{y}_{x}=2 v$, such that $v$ satisfies the $\mathrm{mKdV}$ hierarchy, i.e.

$$
\frac{\partial}{\partial x}\left(\frac{\partial}{\partial x}+2 v\right) \mathcal{L}_{n}\left[x ; 2\left(v_{x}-v^{2}\right)\right]+2 v_{t}=0
$$

then expression (8) yields the following condition on $\phi$

$$
\frac{\partial}{\partial x}\left(-2 \frac{\phi_{x}}{\phi} \mathcal{L}_{n}\left[x ; \tilde{y}_{x x}-\frac{1}{2} \tilde{y}_{x}^{2}\right]\right)+\frac{d}{d t}\left(-2 \frac{\phi_{x}}{\phi}\right)+\beta(t) \frac{\phi_{x}}{\phi^{2}}=0
$$

By integrating the above equation in $x$, we have

$$
2 \phi_{x} \mathcal{L}_{n}\left[x ; \tilde{y}_{x x}-\frac{1}{2} \tilde{y}_{x}^{2}\right]+2 \phi_{t}+\beta(t)+\alpha(t) \phi=0
$$

where $\alpha(t)$ is an arbitrary function. Condition (11) is similar to one found in [23] for the $\mathrm{mKdV}$ equation, although it was not derived any explicit solutions there.

In order to check that the equation (11) is compatible with the assumption (9), let us isolate $\mathcal{L}_{n}\left[x ; \tilde{y}_{x x}-\frac{1}{2} \tilde{y}_{x}^{2}\right]$, i. e.

$$
\mathcal{L}_{n}\left[x ; \tilde{y}_{x x}-\frac{1}{2} \tilde{y}_{x}^{2}\right]=-\frac{\phi_{t}}{\phi_{x}}-\frac{(\beta(t)+\alpha(t) \phi)}{2 \phi_{x}}
$$

The above expression together with the definition $\tilde{y}=\ln \left[\phi_{x}\right]$ yields

$$
\begin{gathered}
\frac{\partial}{\partial x} \mathcal{L}_{n}\left[x ; \tilde{y}_{x x}-\frac{1}{2} \tilde{y}_{x}^{2}\right]=-\frac{\phi_{x t}}{\phi_{x}}+\frac{\phi_{t} \phi_{x x}}{\phi_{x}^{2}}+\frac{(\beta(t)+\alpha(t) \phi) \phi_{x x}}{2 \phi_{x}^{2}}-\frac{\alpha(t)}{2} \\
\tilde{y}_{x} \mathcal{L}_{n}\left[x ; \tilde{y}_{x x}-\frac{1}{2} \tilde{y}_{x}^{2}\right]=-\frac{\phi_{t} \phi_{x x}}{\phi_{x}^{2}}-\frac{(\beta(t)+\alpha(t) \phi) \phi_{x x}}{2 \phi_{x}^{2}}
\end{gathered}
$$

By summing (13) and (14), we have

$$
\left(\frac{\partial}{\partial x}+\tilde{y}_{x}\right) \mathcal{L}_{n}\left[x ; \tilde{y}_{x x}-\frac{1}{2} \tilde{y}_{x}^{2}\right]+\tilde{y}_{t}+\frac{\alpha(t)}{2}=0
$$

which gives (9) through a derivation and the transformation $\tilde{y}_{x}=2 v$. Hence $\tilde{y}$ is given in terms of $v$ by

$$
\tilde{y}=2 \int^{x} v\left(x^{\prime}, t\right) d x^{\prime}+\Gamma(t)
$$


where $\Gamma(t)$ is an arbitrary function. If we consider a soliton solution for $v$, we must choose $\alpha(t)=-2 \Gamma^{\prime}(t)$. This can be verified by substituting (16) in (9). Thus, we can rewrite equation (11) as

$$
\frac{\partial}{\partial t}\left(e^{-\Gamma(t)} \phi\right)+\frac{\beta(t) e^{-\Gamma(t)}}{2}=-e^{\tilde{y}-\Gamma(t)} \mathcal{L}_{n}\left[x ; \tilde{y}_{x x}-\frac{1}{2} \tilde{y}_{x}^{2}\right]
$$

As the right hand side of the equation (17) is expressed in terms of known elements, we can integrate it in order to determine $\phi$, i.e.

$$
\phi=-e^{\Gamma(t)}\left(\int_{-\infty}^{t} e^{\tilde{y}-\Gamma\left(t^{\prime}\right)} \mathcal{L}_{n}\left[x ; \tilde{y}_{x x}-\frac{1}{2} \tilde{y}_{x}^{2}\right] d t^{\prime}+\int_{-\infty}^{t} \frac{\beta\left(t^{\prime}\right) e^{-\Gamma\left(t^{\prime}\right)}}{2} d t^{\prime}+\Phi_{0}(x)\right)
$$

where $\Phi_{0}(x)$ must be determined for $(18)$ to be consistent with the definition $\tilde{y}=\ln \phi_{x}$. Let us derive (18) in $x$ and use (15) such that

$\frac{\partial \phi}{\partial x}=-e^{\Gamma(t)}\left(\int_{-\infty}^{t} e^{\tilde{y}-\Gamma\left(t^{\prime}\right)}\left(\frac{\partial}{\partial x}+\tilde{y}_{x}\right) \mathcal{L}_{n}\left[x ; \tilde{y}_{x x}-\frac{1}{2} \tilde{y}_{x}^{2}\right] d t^{\prime}+\Phi_{0}^{\prime}(x)\right)=e^{\tilde{y}}-e^{\Gamma(t)}\left(\lim _{t^{\prime} \rightarrow-\infty} e^{\tilde{y}\left(x, t^{\prime}\right)-\Gamma\left(t^{\prime}\right)}+\Phi_{0}^{\prime}(x)\right)$.

By using the definition of $\tilde{y}$, the above expression yields the condition

$$
\Phi_{0}(x)=-\int^{x} \lim _{t^{\prime} \rightarrow-\infty} e^{\tilde{y}\left(x^{\prime}, t^{\prime}\right)-\Gamma\left(t^{\prime}\right)} d x^{\prime}+c_{1}, \quad c_{1} \equiv \text { constant }
$$

Observe that a soliton solution from the mKdV hierarchy yields $\Phi_{0}(x)=-x+c_{1}$. Let us see three examples that work for the whole mKdV-Liouville hierarchy:

Example 1) The vacuum solution of the mKdV hierarchy, i. e. $v=0$ yields $\tilde{y}=\Gamma(t)$ and

$$
\begin{gathered}
\phi=e^{\Gamma(t)}\left(-\int^{t} \frac{\beta\left(t^{\prime}\right) e^{-\Gamma\left(t^{\prime}\right)}}{2} d t^{\prime}+x-c_{1}\right) \\
y=-2 \ln \left(-\int^{t} \frac{\beta\left(t^{\prime}\right) e^{-\Gamma\left(t^{\prime}\right)}}{2} d t^{\prime}+x-c_{1}\right)-\Gamma(t)
\end{gathered}
$$

Example 2) The 1-soliton solution of the mKdV hierarchy, i. e.

$$
v=\frac{\partial}{\partial x} \ln \left(\frac{2-e^{\eta}}{2+e^{\eta}}\right) \quad \eta=k x-k^{2 n+1} t,
$$

yields

$$
\begin{gathered}
\phi=e^{\Gamma(t)}\left(-\frac{4 e^{\eta}}{k\left(2+e^{\eta}\right)}-\int^{t} \frac{\beta\left(t^{\prime}\right) e^{-\Gamma\left(t^{\prime}\right)}}{2} d t^{\prime}+x-c_{1}\right) \\
y=2 \ln \left(\frac{2-e^{\eta}}{2+e^{\eta}}\right)-2 \ln \left(-\frac{4 e^{\eta}}{k\left(2+e^{\eta}\right)}-\int^{t} \frac{\beta\left(t^{\prime}\right) e^{-\Gamma\left(t^{\prime}\right)}}{2} d t^{\prime}+x-c_{1}\right)-\Gamma(t)
\end{gathered}
$$

Example 3) The 2-soliton solution of the mKdV hierarchy, i. e

$$
v=\frac{\partial}{\partial x} \ln \left(\frac{4+2\left(e^{\eta_{1}}+e^{\eta_{2}}\right)+\left(\frac{k_{1}-k_{2}}{k_{1}+k_{2}}\right)^{2} e^{\eta_{1}+\eta_{2}}}{4-2\left(e^{\eta_{1}}+e^{\eta_{2}}\right)+\left(\frac{k_{1}-k_{2}}{k_{1}+k_{2}}\right)^{2} e^{\eta_{1}+\eta_{2}}}\right), \quad \eta_{j}=k_{j} x-k_{j}^{2 n+1} t, \quad j=1,2
$$


yields

$$
\begin{aligned}
\phi & =e^{\Gamma(t)}\left(-\frac{4\left(k_{1}+k_{2}\right)}{k_{1} k_{2}}\left(\frac{k_{1}^{2} e^{\eta_{2}}\left(e^{\eta_{1}}-2\right)-2 k_{1} k_{2}\left(e^{\eta_{1}}+e^{\eta_{2}}+e^{\eta_{1}+\eta_{2}}\right)+k_{2}^{2} e^{\eta_{1}}\left(e^{\eta_{2}}-2\right)}{k_{1}^{2}\left(e^{\eta_{1}}-2\right)\left(e^{\eta_{2}}-2\right)-2 k_{1} k_{2}\left(2 e^{\eta_{1}}+2 e^{\eta_{2}}+e^{\eta_{1}+\eta_{2}}-4\right)+k_{2}^{2}\left(e^{\eta_{1}}-2\right)\left(e^{\eta_{2}}-2\right)}\right)\right. \\
& \left.-\int^{t} \frac{\beta\left(t^{\prime}\right) e^{-\Gamma\left(t^{\prime}\right)}}{2} d t^{\prime}+x-c_{1}\right) \\
y & =2 \ln \left(\frac{4+2\left(e^{\eta_{1}}+e^{\eta_{2}}\right)+\left(\frac{k_{1}-k_{2}}{k_{1}+k_{2}}\right)^{2} e^{\eta_{1}+\eta_{2}}}{4-2\left(e^{\eta_{1}}+e^{\eta_{2}}\right)+\left(\frac{k_{1}-k_{2}}{k_{1}+k_{2}}\right)^{2} e^{\eta_{1}+\eta_{2}}}\right) \\
& -2 \ln \left(-\frac{4\left(k_{1}+k_{2}\right)}{k_{1} k_{2}}\left(\frac{k_{1}^{2} e^{\eta_{2}}\left(e^{\eta_{1}}-2\right)-2 k_{1} k_{2}\left(e^{\eta_{1}}+e^{\eta_{2}}+e^{\eta_{1}+\eta_{2}}\right)+k_{2}^{2} e^{\eta_{1}}\left(e^{\eta_{2}}-2\right)}{k_{1}^{2}\left(e^{\eta_{1}}-2\right)\left(e^{\eta_{2}}-2\right)-2 k_{1} k_{2}\left(2 e^{\eta_{1}}+2 e^{\eta_{2}}+e^{\eta_{1}+\eta_{2}}-4\right)+k_{2}^{2}\left(e^{\eta_{1}}-2\right)\left(e^{\eta_{2}}-2\right)}\right)\right. \\
& \left.-\int^{t} \frac{\beta\left(t^{\prime}\right) e^{-\Gamma\left(t^{\prime}\right)}}{2} d t^{\prime}+x-c_{1}\right)-\Gamma(t)
\end{aligned}
$$

If we choose $\beta(t)=0$, the results of this section represent a map from the $\mathrm{mKdV}$ hierarchy into itself. By defining the field $\tilde{v}=y_{x} / 2$, we have solutions for the $\mathrm{mKdV}$ hierarchy which appears to be new to the author knowledge. Below, we show the solutions for the $\mathrm{mKdV}$ equation which can be obtained from the examples we have just used:

Example 1) $\quad \tilde{v}=\frac{1}{c_{1}-x}$

Example 2) $\quad \tilde{v}=\frac{k\left[4+4 e^{\eta}\left(k\left(x-c_{1}\right)-2\right)-e^{2 \eta}\right]}{4 k\left(c_{1}-x\right)+8 e^{\eta}+e^{2 \eta}\left(k\left(x-c_{1}\right)-4\right)}$

Example 3) $\tilde{v}=\left[-4+4 e^{\eta_{1}}\left(k_{1}\left(x-c_{1}\right)-2\right)+4 e^{\eta_{2}}\left(k_{2}\left(x-c_{1}\right)-2\right)+e^{2 \eta_{1}}+e^{2 \eta_{2}}\right.$

$$
\begin{aligned}
& +\frac{8\left(k_{1}^{4}-k_{1}^{2} k_{2}^{2}+k_{2}^{4}\right)}{k_{1} k_{2}\left(k_{1}+k_{2}\right)^{2}} e^{\eta_{1}+\eta_{2}}-\frac{\left(k_{1}^{2}-k_{2}^{2}\right)^{2}}{k_{1} k_{2}\left(k_{1}+k_{2}\right)^{2}}\left[k_{1} e^{\eta_{1}+2 \eta_{2}}\left(k_{1} k_{2}\left(x-c_{1}\right)-4 k_{1}-2 k_{2}\right)\right. \\
& \left.\left.+k_{2} e^{2 \eta_{1}+\eta_{2}}\left(k_{1} k_{2}\left(x-c_{1}\right)-2 k_{1}-4 k_{2}\right)\right]-\frac{\left(k_{1}-k_{2}\right)^{4}}{4\left(k_{1}+k_{2}\right)^{4}} e^{2\left(\eta_{1}+\eta_{2}\right)}\right] /\left[4\left(x-c_{1}\right)\right. \\
& +\frac{8 e^{\eta_{1}}}{k_{1}}+\frac{8 e^{\eta_{2}}}{k_{2}}+e^{2 \eta_{1}}\left(c_{1}-x+\frac{4}{k_{1}}\right)+e^{2 \eta_{2}}\left(c_{1}-x+\frac{4}{k_{2}}\right) \\
& +\frac{8 k_{1} k_{2}\left(c_{1}-x\right)+2\left(k_{1}+k_{2}\right)}{\left(k_{1}+k_{2}\right)^{2}} e^{\eta_{1}+\eta_{2}}-\frac{2\left(k_{1}-k_{2}\right)^{2}}{k_{1}\left(k_{1}+k_{2}\right)^{2}} e^{\eta_{1}+2 \eta_{2}} \\
& \left.-\frac{2\left(k_{1}-k_{2}\right)^{2}}{k_{2}\left(k_{1}+k_{2}\right)^{2}} e^{2 \eta_{1}+\eta_{2}}+\frac{\left(k_{1}-k_{2}\right)^{4}\left(k_{1} k_{2}\left(x-c_{1}\right)-4\left(k_{1}+k_{2}\right)\right)}{4 k_{1} k_{2}\left(k_{1}+k_{2}\right)^{4}} e^{2\left(\eta_{1}+\eta_{2}\right)}\right]
\end{aligned}
$$

\subsection{Self-similarity reduction}

Let us consider the expression (9) again. By theorem 2 of the appendix, we always can choose

$$
\beta(t)=\beta_{0}[(2 n+1) t]^{-(2 n+2) /(2 n+1)} .
$$




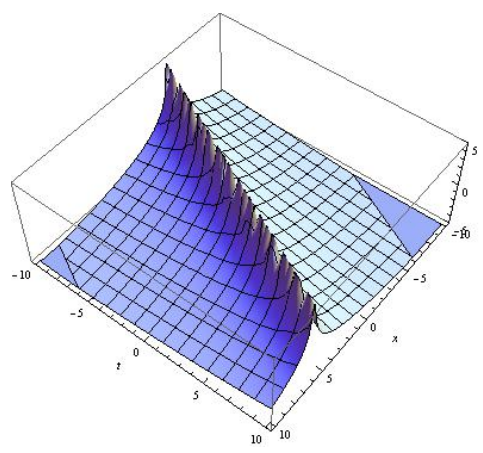

(a) Example 1

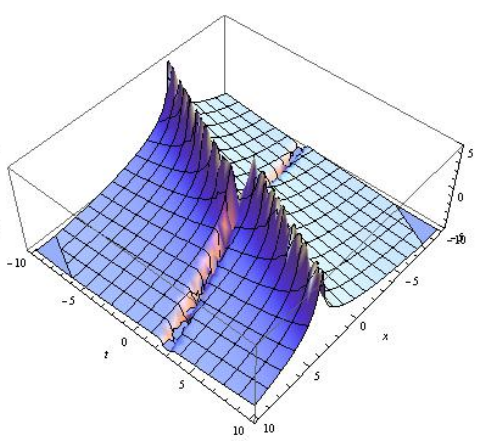

(b) Example 2

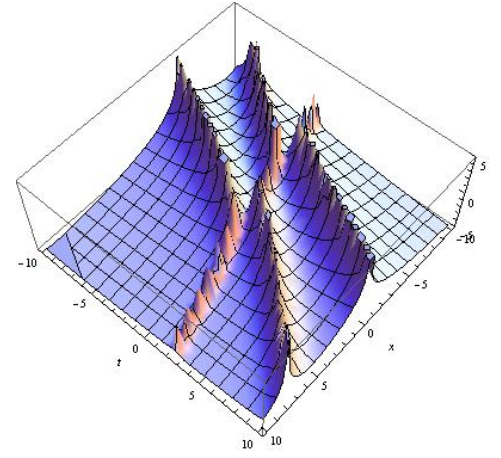

(c) Example 3

Figure 1: It was showed we can obtain solutions for the mKdV-Liouville hierarchy from the solutions of the $\mathrm{mKdV}$ hierarchy. In these figures, we illustrate some solutions for the first equation of the mKdV-Liouville hierarchy $(n=1)$ with $\beta(t)=1$. Figure $1 \mathrm{a}$ shows solution (20) with $\Gamma(t)=0$ and $c_{1}=1$. Figure $1 \mathrm{~b}$ shows solution 21 with $\Gamma(t)=0$, $c_{1}=1$ and $k=-2$. Figure $1 \mathrm{c}$ shows solution 22 with $\Gamma(t)=0, c_{1}=1, k_{1}=-1$ and $k_{2}=-2$.

Thus, by assuming the self-similarity reduction

$$
z=x[(2 n+1) t]^{-1 /(2 n+1)} \quad y(x, t)=y(z), \quad \mathcal{L}_{k}\left[x ; y_{x x}-\frac{1}{2} y_{x}^{2}\right]=[(2 n+1) t]^{-2 k /(2 n+1)} \mathcal{L}_{k}\left[z ; y_{z z}-\frac{1}{2} y_{z}^{2}\right],
$$

the mKdV-Liouville hierarchy reduce to

$$
\frac{\partial}{\partial z}\left(\frac{\partial}{\partial z}+y_{z}\right) \mathcal{L}_{n}\left[z ; y_{z z}-\frac{1}{2} y_{z}^{2}\right]-\left(z y_{z}\right)_{z}+\beta_{0} e^{y}=0
$$

Hierarchy 23) is a particular case of the hierarchy proposed in [8]. If we choose $g(t)=[(2 n+1) t]^{1 /(2 n+1)}$, the definition (6) reduce to

$$
\tilde{y}(x, t)=\tilde{y}(z), \quad \phi(x, t)=\phi(z)
$$

Thus, the self-similarity reduction of (8) yields

$$
\frac{\partial}{\partial z}\left(\frac{\partial}{\partial z}+\tilde{y}_{z}-2 \frac{\phi_{z}}{\phi}\right) \mathcal{L}_{n}\left[z ; \tilde{y}_{z z}-\frac{1}{2} \tilde{y}_{z}^{2}\right]-\frac{d}{d z}\left(z \tilde{y}_{z}-2 z \frac{\phi_{z}}{\phi}\right)+\beta_{0} \frac{\phi_{z}}{\phi^{2}}=0
$$

which can be integrated and the integration constant defined as $2(\alpha-1)$, i. e.

$$
\left(\frac{\partial}{\partial z}+\tilde{y}_{z}-2 \frac{\phi_{z}}{\phi}\right) \mathcal{L}_{n}\left[z ; \tilde{y}_{z z}-\frac{1}{2} \tilde{y}_{z}^{2}\right]-z \tilde{y}_{z}+2 z \frac{\phi_{z}}{\phi}-\frac{\beta_{0}}{\phi}+2(\alpha-1)=0
$$

Let us assume that $\tilde{y}$ satisfies

$$
\left(\frac{\partial}{\partial z}+\tilde{y}_{z}\right) \mathcal{L}_{n}\left[z ; \tilde{y}_{z z}-\frac{1}{2} \tilde{y}_{z}^{2}\right]-z \tilde{y}_{z}-2 \alpha=0,
$$

such that 25 is simplified to

$$
-2 \frac{\phi_{z}}{\phi} \mathcal{L}_{n}\left[z ; \tilde{y}_{z z}-\frac{1}{2} \tilde{y}_{z}^{2}\right]+2 z \frac{\phi_{z}}{\phi}-\frac{\beta_{0}}{\phi}+2(2 \alpha-1)=0
$$


In order to check the compatibility between 26 and 27 , let us isolate $\mathcal{L}_{n}\left[z ; \tilde{y}_{z z}-\right.$ $\left.\frac{1}{2} \tilde{y}_{z}^{2}\right]$, i.e.

$$
\mathcal{L}_{n}\left[z ; \tilde{y}_{z z}-\frac{1}{2} \tilde{y}_{z}^{2}\right]=z-\frac{\beta_{0}}{2 \phi_{z}}+(2 \alpha-1) \frac{\phi}{\phi_{z}}
$$

such that

$$
\begin{aligned}
& \frac{\partial}{\partial z} \mathcal{L}_{n}\left[z ; \tilde{y}_{z z}-\frac{1}{2} \tilde{y}_{z}^{2}\right]=\frac{\beta_{0} \phi_{z z}}{2 \phi_{z}^{2}}+2 \alpha-(2 \alpha-1) \frac{\phi \phi_{z z}}{\phi_{z}^{2}} \\
& \tilde{y}_{z} \mathcal{L}_{n}\left[z ; \tilde{y}_{z z}-\frac{1}{2} \tilde{y}_{z}^{2}\right]=z \tilde{y}_{z}-\frac{\beta_{0} \phi_{z z}}{2 \phi_{z}^{2}}+(2 \alpha-1) \frac{\phi \phi_{z z}}{\phi_{z}^{2}}
\end{aligned}
$$

It is trivial to check that $(26)$ is true with the above expressions. The transformation

$$
\tilde{y}=2 \int^{z} v\left(z^{\prime}\right) d z^{\prime}+C
$$

maps the hierarchy 26 into the the PII hierarchy, i. e.

$$
\left(\frac{\partial}{\partial z}+2 v\right) \mathcal{L}_{n}\left[z ; 2\left(v_{z}-v^{2}\right)\right]-2 z v-2 \alpha=0
$$

which has the PII equation as the first equation, namely

$$
v_{z z}=2 v^{3}+z v+\alpha .
$$

From (27), we have

$$
\phi=\frac{e^{\tilde{y}}\left(\mathcal{L}_{n}\left[z ; \tilde{y}_{z z}-\frac{1}{2} \tilde{y}_{z}^{2}\right]-z\right)+\beta_{0} / 2}{(2 \alpha-1)}
$$

provided that $\alpha \neq 1 / 2$. Therefore, the solution for $(23)$ is related with the solution of the PII hierarchy by the transformation

$$
y=2 \int^{z} v\left(z^{\prime}\right) d z^{\prime}+C+2 \ln (2 \alpha-1)-2 \ln \left[e^{\tilde{y}}\left(\mathcal{L}_{n}\left[z ; 2\left(v_{z}-v^{2}\right)\right]-z\right)+\beta_{0} / 2\right]
$$

Observe that the first equation of the hierarchy (23) is a forth order equation, i.e.

$$
y_{z z z z}-\frac{3}{2} y_{z}^{2} y_{z z}-z y_{z z}-y_{z}+\beta_{0} e^{y}=0
$$

which is a particular case of the equation proposed in [8] as a possibility to define a new transcendental function.

The general solution of the PII equation define a transcendental solution with two arbitrary constants plus the parameter $\alpha$. The solution $(30)$ for $n=1$ has the arbitrary constants $C, \alpha$ and two constants of integration from the general solution of the PII equation. Thus, solution (30) represent the general solution for the equation (31), which is a particular case of the Kudryashov's equation.

As examples, we show some solutions for equation (31) based on rational solutions for the PII equation in table 1 . 
Table 1: Solutions for equation (31) based on rational solutions from PII equation

\begin{tabular}{|l|l|l|}
\hline$\alpha=-2$ & $v=\frac{2\left(z^{3}-2\right)}{z\left(z^{3}+4\right)}$ & $y=C-2 \ln \left(\frac{2 e^{C}\left(z^{6}+20 z^{3}-80\right)-\beta_{0} z}{10\left(z^{3}+4\right)}\right)$ \\
\hline$\alpha=-1$ & $v=\frac{1}{z}$ & $y=C-2 \ln \left(\frac{2 e^{C}\left(z^{3}+4\right)-\beta}{6 z}\right)$ \\
\hline$\alpha=0$ & $v=0$ & $y=C-2 \ln \left(e^{C} z-\frac{\beta_{0}}{2}\right)$ \\
\hline$\alpha=1$ & $v=-\frac{1}{z}$ & $y=C-2 \ln \left(\frac{\beta_{0} z}{2}-e^{C}\right)$ \\
\hline$\alpha=2$ & $v=-\frac{2\left(z^{3}-2\right)}{z\left(z^{3}+4\right)}$ & $y=C-2 \ln \left(\frac{\beta_{0}\left(z^{3}+4\right)-2 e^{C}}{6 z}\right)$ \\
\hline
\end{tabular}

\section{Conclusion}

In this paper, it was shown the relation between the mKdV and mKdV-Liouville hierarchies by using an extension of the modified truncation approach. Some solutions for the mKdV-Liouville was presented, such that a particular case of these solutions yields new solutions for the mKdV hierarchy. Also, it was showed the general solution for a particular case of the Kudryashov's equation in terms of the second Painlevé transcendent.

\section{Acknowledgements}

I am thankful to J. F. Gomes and A. H. Zimerman for discussions. The author thanks FAPESP (2010/18110-9) for financial support.

\section{Appendix A. Properties of the generalized mKdV-sinh-Gordon hierarchy}

Consider the generalized mKdV-sinh-Gordon hierarchy

$$
E_{n}\left(y ; \epsilon_{0}(t), \beta(t), \delta(t)\right): \quad \epsilon_{0}(t) \frac{\partial}{\partial x}\left(\frac{\partial}{\partial x}+y_{x}\right) \mathcal{L}_{n}\left[x ; y_{x x}-\frac{1}{2} y_{x}^{2}\right]+y_{x t}+\beta(t) e^{y}+\delta(t) e^{-y}=0
$$

where $\mathcal{L}_{n}[x ; u]$ is the Lenard recurrence relation, i.e.

$$
\frac{\partial}{\partial x} \mathcal{L}_{n+1}[x ; u]=\left(\frac{\partial^{3}}{\partial x^{3}}+2 u \frac{\partial}{\partial x}+u_{x}\right) \mathcal{L}_{n}[x ; u], \quad \mathcal{L}_{0}[x ; u]=1 .
$$

In this appendix we show that the generalized mKdV-sinh-Gordon hierarchy can be reduced in two simpler cases, namely, the mKdV-sinh-Gordon hierarchy or the mKdVLiouville hierarchy. In order to show this, let us divide $\mathcal{L}_{n}\left[x ; y_{x x}-\frac{1}{2} y_{x}^{2}\right]$ in two parts as

$$
\mathcal{L}_{n}\left[x ; y_{x x}-\frac{1}{2} y_{x}^{2}\right]=\mathcal{L}_{n}^{(e)}\left[x ; y_{x x}-\frac{1}{2} y_{x}^{2}\right]+\mathcal{L}_{n}^{(o)}\left[x ; y_{x x}-\frac{1}{2} y_{x}^{2}\right]
$$


where we define $\mathcal{L}_{n}^{(e)}\left[x ; y_{x x}-\frac{1}{2} y_{x}^{2}\right]$ and $\mathcal{L}_{n}^{(o)}\left[x ; y_{x x}-\frac{1}{2} y_{x}^{2}\right]$ as the parts of $\mathcal{L}_{n}\left[x ; y_{x x}-\frac{1}{2} y_{x}^{2}\right]$ with even and odd dimension of the field respectively. For example

$\mathcal{L}_{1}\left[x ; y_{x x}-\frac{1}{2} y_{x}^{2}\right]=y_{x x}-\frac{1}{2} y_{x}^{2} \quad \Rightarrow \quad \mathcal{L}_{1}^{(e)}\left[x ; y_{x x}-\frac{1}{2} y_{x}^{2}\right]=-\frac{1}{2} y_{x}^{2}, \quad \mathcal{L}_{1}^{(o)}\left[x ; y_{x x}-\frac{1}{2} y_{x}^{2}\right]=y_{x x}$.

From the definition of equation A.2), observe that

$$
\begin{aligned}
\mathcal{L}_{n}^{(e)}\left[x ; y_{x x}-\frac{1}{2} y_{x}^{2}\right] & =\mathcal{L}_{n}^{(e)}\left[x ;-y_{x x}-\frac{1}{2} y_{x}^{2}\right] \\
\mathcal{L}_{n}^{(o)}\left[x ; y_{x x}-\frac{1}{2} y_{x}^{2}\right] & =-\mathcal{L}_{n}^{(o)}\left[x ;-y_{x x}-\frac{1}{2} y_{x}^{2}\right]
\end{aligned}
$$

Hence, the Lenard recurrence relation is equivalent to the following system

$$
\begin{aligned}
\frac{\partial}{\partial x} \mathcal{L}_{n+1}^{(e)}\left[x ; y_{x x}-\frac{1}{2} y_{x}^{2}\right] & =\left(\frac{\partial^{3}}{\partial x^{3}}-y_{x}^{2} \frac{\partial}{\partial x}-\frac{1}{2}\left(y_{x}^{2}\right)_{x}\right) \mathcal{L}_{n}^{(e)}\left[x ; y_{x x}-\frac{1}{2} y_{x}^{2}\right]+\left(2 y_{x x} \frac{\partial}{\partial x}+y_{x x x}\right) \mathcal{L}_{n}^{(o)}\left[x ; y_{x x}-\frac{1}{2} y_{x}^{2}\right] \\
\frac{\partial}{\partial x} \mathcal{L}_{n+1}^{(o)}\left[x ; y_{x x}-\frac{1}{2} y_{x}^{2}\right] & =\left(\frac{\partial^{3}}{\partial x^{3}}-y_{x}^{2} \frac{\partial}{\partial x}-\frac{1}{2}\left(y_{x}^{2}\right)_{x}\right) \mathcal{L}_{n}^{(o)}\left[x ; y_{x x}-\frac{1}{2} y_{x}^{2}\right]+\left(2 y_{x x} \frac{\partial}{\partial x}+y_{x x x}\right) \mathcal{L}_{n}^{(e)}\left[x ; y_{x x}-\frac{1}{2} y_{x}^{2}\right]
\end{aligned}
$$

With the above properties, we can proof the following auto-Bäcklund transformation.

Theorem 1. Let $y=y(x, t)$ be the solution of $E_{n}\left(y ; \epsilon_{0}(t), \beta(t), \delta(t)\right)$, then $\tilde{y}=-y$ is the solution of $E_{n}\left(\tilde{y} ; \epsilon_{0}(t),-\delta(t),-\beta(t)\right)$.

Proof. Consider the hierarchy

$E_{n}\left(\tilde{y} ; \tilde{\epsilon}_{0}(t), \tilde{\beta}(t), \tilde{\delta}(t)\right): \quad \tilde{\epsilon}_{0}(t) \frac{\partial}{\partial x}\left(\frac{\partial}{\partial x}+\tilde{y}_{x}\right) \mathcal{L}_{n}\left[x ; \tilde{y}_{x x}-\frac{1}{2} \tilde{y}_{x}^{2}\right]+\tilde{y}_{x t}+\tilde{\beta}(t) e^{\tilde{y}}+\tilde{\delta}(t) e^{-\tilde{y}}=0$

By the transformation $\tilde{y}=-y$, we have

$$
\tilde{\epsilon}_{0}(t) \frac{\partial}{\partial x}\left(\frac{\partial}{\partial x}-y_{x}\right) \mathcal{L}_{n}\left[x ;-y_{x x}-\frac{1}{2} y_{x}^{2}\right]-y_{x t}+\tilde{\beta}(t) e^{-y}+\tilde{\delta}(t) e^{y}=0
$$

Let us assume $\tilde{\epsilon}_{0}(t)=\epsilon_{0}(t) \neq 0, \tilde{\beta}(t)=-\delta(t)$ and $\tilde{\delta}(t)=-\beta(t)$. In order to proof that $y$ satisfy (1), we need to proof that

$$
\left(\frac{\partial}{\partial x}-y_{x}\right) \mathcal{L}_{n}\left[x ;-y_{x x}-\frac{1}{2} y_{x}^{2}\right]=-\left(\frac{\partial}{\partial x}+y_{x}\right) \mathcal{L}_{n}\left[x ; y_{x x}-\frac{1}{2} y_{x}^{2}\right]
$$

Observe we can rewrite equation (A.6) as

$$
\frac{\partial}{\partial x} \mathcal{L}_{n}^{(e)}\left[x ; y_{x x}-\frac{1}{2} y_{x}^{2}\right]+y_{x} \mathcal{L}_{n}^{(o)}\left[x ; y_{x x}-\frac{1}{2} y_{x}^{2}\right]=0
$$

By deriving twice the above equation, we have

$\frac{\partial^{3}}{\partial x^{3}} \mathcal{L}_{n}^{(e)}\left[x ; y_{x x}-\frac{1}{2} y_{x}^{2}\right]+y_{x x x} \mathcal{L}_{n}^{(o)}\left[x ; y_{x x}-\frac{1}{2} y_{x}^{2}\right]+2 y_{x x} \frac{\partial}{\partial x} \mathcal{L}_{n}^{(o)}\left[x ; y_{x x}-\frac{1}{2} y_{x}^{2}\right]+y_{x} \frac{\partial^{2}}{\partial x^{2}} \mathcal{L}_{n}^{(o)}\left[x ; y_{x x}-\frac{1}{2} y_{x}^{2}\right]=0$ 
Expression A.7 is verified by induction. It is ease to verify that A.7 is true for $n=0$ and $n=1$, i.e.

$$
\begin{gathered}
\mathcal{L}_{0}\left[x ; y_{x x}-\frac{1}{2} y_{x}^{2}\right]=1 \quad \Rightarrow \quad \mathcal{L}_{0}^{(e)}\left[x ; y_{x x}-\frac{1}{2} y_{x}^{2}\right]=1, \quad \mathcal{L}_{0}^{(o)}\left[x ; y_{x x}-\frac{1}{2} y_{x}^{2}\right]=0 \\
\mathcal{L}_{1}\left[x ; y_{x x}-\frac{1}{2} y_{x}^{2}\right]=y_{x x}-\frac{1}{2} y_{x}^{2} \quad \Rightarrow \quad \mathcal{L}_{1}^{(e)}\left[x ; y_{x x}-\frac{1}{2} y_{x}^{2}\right]=-\frac{1}{2} y_{x}^{2}, \quad \mathcal{L}_{1}^{(o)}\left[x ; y_{x x}-\frac{1}{2} y_{x}^{2}\right]=y_{x x} .
\end{gathered}
$$

Now, let us assume that expression A.7) is true for $n=k-1$. Using (A.3) and (A.4) in expression A.7 with $n=k$ yields

$$
\begin{aligned}
& \left(\frac{\partial^{3}}{\partial x^{3}}-y_{x}^{2} \frac{\partial}{\partial x}-\frac{1}{2}\left(y_{x}^{2}\right)_{x}\right) \mathcal{L}_{k-1}^{(e)}\left[x ; y_{x x}-\frac{1}{2} y_{x}^{2}\right]+\left(2 y_{x x} \frac{\partial}{\partial x}+y_{x x x}\right) \mathcal{L}_{k-1}^{(o)}\left[x ; y_{x x}-\frac{1}{2} y_{x}^{2}\right]+ \\
& y_{x}\left\{\left(\frac{\partial^{2}}{\partial x^{2}}-y_{x}^{2}\right) \mathcal{L}_{k-1}^{(o)}\left[x ; y_{x x}-\frac{1}{2} y_{x}^{2}\right]+y_{x x} \mathcal{L}_{k-1}^{(e)}\left[x ; y_{x x}-\frac{1}{2} y_{x}^{2}\right]+\right. \\
& \left.\int^{x} y_{x x}\left(\frac{\partial}{\partial x} \mathcal{L}_{k-1}^{(e)}\left[x ; y_{x x}-\frac{1}{2} y_{x}^{2}\right]+y_{x} \mathcal{L}_{k-1}^{(o)}\left[x ; y_{x x}-\frac{1}{2} y_{x}^{2}\right]\right) d x\right\}=0
\end{aligned}
$$

Therefore, by using (A.7) and A.8 with $n=k-1$, the above equation is verified.

Theorem 2. Let $y=y(x, t)$ be the solution of $E_{n}\left(y ; \epsilon_{0}(t), \beta(t), \delta(t)\right)$, then the transformation

$$
\tilde{y}=y(x, \tilde{t})+\ln f(\tilde{t}), \quad \tilde{t}=\int^{t} \frac{d t^{\prime}}{\epsilon_{0}\left(t^{\prime}\right)}
$$

gives the solution for $E_{n}\left(\tilde{y} ; 1, \frac{\beta(t) f(\tilde{t})}{\epsilon_{0}(t)}, \frac{\delta(t)}{f(\tilde{t}) \epsilon_{0}(t)}\right)$, with $t=t(\tilde{t})$, provided that $\epsilon_{0}(t) \neq 0$ and $f(\tilde{t}) \neq 0$.

Proof. It is a direct substitution.

Using theorem 2 with $f(\tilde{t})= \pm i \sqrt{\frac{\delta(t)}{\beta(t)}}$, we can reduce the hierarchy A.1, with $\epsilon_{0}(t)$, $\beta(t)$ and $\delta(t)$ non-null, to the usual mKdV-sinh-Gordon hierarchy

$$
\frac{\partial}{\partial x}\left(\frac{\partial}{\partial x}+\tilde{y}_{x}\right) \mathcal{L}_{n}\left[x ; \tilde{y}_{x x}-\frac{1}{2} \tilde{y}_{x}^{2}\right]+\tilde{y}_{x \tilde{t}}+\eta(\tilde{t}) \sinh \tilde{y}=0, \quad \eta(\tilde{t})= \pm \frac{2 i \sqrt{\delta(t) \beta(t)}}{\epsilon_{0}(t)}
$$

The hierarchy A.1 with $\delta(t)=0$ is the mixed mKdV-Liouville hierarchy and, by theorem 2, can be reduced to

$$
\frac{\partial}{\partial x}\left(\frac{\partial}{\partial x}+\tilde{y}_{x}\right) \mathcal{L}_{n}\left[x ; \tilde{y}_{x x}-\frac{1}{2} \tilde{y}_{x}^{2}\right]+\tilde{y}_{x \tilde{t}}+\tilde{\beta}(\tilde{t}) e^{\tilde{y}}=0, \quad \tilde{\beta}(\tilde{t})=\frac{\beta(t) f(\tilde{t})}{\epsilon_{0}(t)}
$$

Observe that the case $\beta(t)=0$ and $\delta(t) \neq 0$ can be mapped on hierarchy A.10 by theorem 1 . 


\section{References}

[1] G. L. Lamb, Jr., Elements of Soliton Theory (Pure \& Applied Mathematics). John Wiley \& Sons Inc, New York, 1980

[2] Deng-yuan CHEN, Da-jun ZHANG and Shu-fang DENG, The Novel Multi-Solitons of the mKdV-Sine Gordon Equation, J. Phys. Soc. Jpn. 71, no. 2 (2002) 658-659

[3] S. R. Mousavian, H. Jafari, C. M. Khalique and S. A. Karimi, New exact analytical solutions for mKdV equation, TJMCS 2 no. 3 (2011) 413-416

[4] J. F. Gomes, G. S. França, A. H. Zimerman, Nonvanishing boundary condition for the mKdV hierarchy and the Gardner equation, J. Phys. A: Math. Theor. 45 (2012) 015207

[5] K. Konno, W. Kameyama and H. Sanuki, Effect of Weak Dislocation Potential on Nonlinear Wave Propagation in Anharmonic Crystal, J. Phys. Soc. Jpn 37 (1974) $171-176$

[6] H. Leblond and D. Mihalache, Few-optical-cycles solitons: Modified Korteweg-de Vries sine-Gordon equation versus other non-slowly-varying-envelope-approximation models, Phys. Rev. A 79 (2009) 063835

[7] D. V. Ruy and G. R. Melo, Extended mixed AKNS-Lund-Regge model and its selfsimilarity reduction, Discontinuity, Nonlinearity, and Complexity 3(2) (2014) 161168

[8] N. A. Kudryashov, One generalization of the second Painlevé hierarchy, J. Phys. A: Math. Theor. 35 (2002) 93-99

[9] H. Umemura, Painlevé Equations and Classical Functions, Sugaku Expositions 11 (1998) $77-100$

[10] C. M. Cosgrove, Higher-Order Painlevé Equation in the Polynomial Class I. Bureau Symbol P2, Studies in Applied Math. 104 (2000) 1-65

[11] C. M. Cosgrove, Higher-order Painlevé equations in the polynomial class II. Bureau symbol P1, Studies in Applied Math. 106 (2006) 321-413

[12] P. R. Gordoa, N. Joshi and A. Pickering, Second and fourth Painlevé hierarchies and Jimbo-Miwa linear problems, J. of Math Phys. 47 (2006) 073504

[13] N. A. Kudryashov, The first and second Painlevé equations of higher order and some relations between them, Phys. Lett. A 224 (1997) 353-360

[14] N. A. Kudryashov, Two hierarchies of ordinary differential equations and their properties, Phys. Lett. A 252 (1999) 173-179

[15] U. Mugãn and F. Jrad, Painlevé test and the first Painlevé hierarchy, J. Phys. A: Math. Gen. 32 (1999) 7933-7952 
[16] U. Mugãn and F. Jrad, Painlevé Test and Higher Order Differential Equation, J. of Nonlinear Math. Phys. 9 (2002) 282-310

[17] A. H. Sakka, Bäcklund Transformations for First and Second Painlevé Hierarchies, SIGMA 5 (2009) 11 pp.

[18] A. H. Sakka, Linear problems and hierarchies of Painlevé equations, J. Phys. A Theor. 42 (2009) 025210

[19] A. H. Sakka, On special solutions of second and fourth Painlevé hierarchies, Phys. Lett. A 373 (2009) 611-615

[20] N. A. Kudryashov, Fourth-order analogies to the Painlevé equation, J. Phys. A: Math. Gen. 35 (2002) 4617-32

[21] N. A. Kudryashov, D. I. Sinelshchikov, Power and Non-Power Asymptotics of Solutions for Generalizations of the Second and Third Painleve Equations, Vestnik natsional'nogo issledovatel'skogo yadernogo universiteta "MIFI" 2(2) (2013)152-160. (in Russian)

[22] P. A. Clarkson, N. Joshi and A. Pickering, Bäcklund transformation for the second Painlevé hierarchy: a modified truncation approach, Inverse Problems 15 (1999) $175-187$

[23] J. Weiss, The Painlevé property for partial differential equations. II: Bäcklund transformation, Lax pairs, and the Schwarzian derivative, J. Math. Phys. 24 (1983) 140513 